\title{
Noninformative priors for the ratio of the scale parameters in the half logistic distributions
}

\author{
Sang Gil Kang ${ }^{1} \cdot$ Dal Ho Kim² ${ }^{2}$ Woo Dong Lee ${ }^{3}$ \\ ${ }^{1}$ Department of Computer and Data Information, Sangji University \\ ${ }^{2}$ Department of Statistics, Kyungpook National University \\ ${ }^{3}$ Department of Asset Management, Daegu Haany University \\ Received 26 June 2012, revised 14 July 2012, accepted 19 July 2012
}

\begin{abstract}
In this paper, we develop the noninformative priors for the ratio of the scale parameters in the half logistic distributions. We develop the first and second order matching priors. It turns out that the second order matching prior matches the alternative coverage probabilities, and is a highest posterior density matching prior. Also we reveal that the one-at-a-time reference prior and Jeffreys' prior are the second order matching prior. We show that the proposed reference prior matches the target coverage probabilities in a frequentist sense through simulation study, and an example based on real data is given.
\end{abstract}

Keywords: Half logistic distribution, matching prior, reference prior, scale parameter.

\section{Introduction}

The half logistic distribution was introduced by Balakrishnan (1985) as a life testing model with increasing hazard rate. Balakrishnan and Puthenpura (1986) obtained the coefficient of the best linear unbiased estimators for the location and scale parameters based on complete and censored samples. Balakrishnan and Wong (1991) obtained the approximate maximum likelihood estimators for the location and scale parameters. Adatia (1997) derived the approximate best linear unbiased estimators of the parameters. Kang and Park (2005) derived the approximate maximum likelihood estimators of the scale parameter based on multiply type-II censored samples. Kim and Han (2010) obtained the maximum likelihood estimator and Bayes estimator for the scale parameter of the half-logistic distribution based on a progressively type II censored sample assuming a natural conjugate prior. However the problem of comparison of two scale parameters has not been considered yet. Thus we want to develop noninformative priors for inference of the ratio of two scale parameters.

Subjective priors are ideal when sufficient information from past experience, expert opinion or previously collected data exist. However, often even without adequate prior information,

\footnotetext{
${ }^{1}$ Associate professor, Department of Computer and Data Information, Sangji University, Wonju 220-702, Korea.

2 Professor, Department of Statistics, Kyungpook National University, Daegu 702-701, Korea.

${ }^{3}$ Corresponding author: Professor, Department of Asset Management, Daegu Haany University, Kyungsan 712-715, Korea. E-mail: wdlee@dhu.ac.kr
} 
one can use Bayesian techniques efficiently with some noninformative or default priors. Once a noninformative prior is developed, then there is no necessity for exploring the effect of hyper parameters.

The notion of a noninformative prior has attracted much attention in recent years. There are two different notions of noninformative priors. One is a probability matching prior introduced by Welch and Peers (1963) which matches the posterior and frequentist probabilities of confidence intervals. Interest in such priors has been revived with the work of Stein (1985) and Tibshirani (1989). Among others, we may cite the work of Mukerjee and Dey (1993), DiCiccio and Stern (1994), Datta and Ghosh (1995a,b, 1996), Mukerjee and Ghosh (1997).

The other is the reference prior introduced by Bernardo (1979) which maximizes the Kullback-Leibler divergence between the prior and the posterior. Ghosh and Mukerjee (1992), and Berger and Bernardo $(1989,1992)$ give a general algorithm to derive a reference prior by splitting the parameters into several groups according to their order of inferential importance. This approach is very successful in various practical problems (Kang, 2011; Kim et al., 2009). Quite often reference priors satisfy the matching criterion described earlier.

The outline of the remaining sections is as follows. In Section 2, we develop first order and second order probability matching priors. We reveal that the second order matching prior is a highest posterior density (HPD) matching prior or matches the alternative coverage probabilities up to the second order. Also we derive the reference priors for the parameter. It turns out that the one-at-a-time reference prior and Jeffreys' prior are the second order matching prior. Section 3 devotes to show that the propriety of the posterior distribution for the general prior including the reference prior and the matching prior. In Section 4, simulated frequentist coverage probabilities under the proposed prior and an example are given.

\section{The noninformative priors}

Suppose that $X$ and $Y$ are independently distributed random variables according to the half-logistic distribution $\mathcal{H} \mathcal{L}\left(\sigma_{1}\right)$ with the scale parameter $\sigma_{1}$, and the half-logistic distribution $\mathcal{H} \mathcal{L}\left(\sigma_{2}\right)$ with the scale parameter $\sigma_{2}$. Then the probability density functions of half logistic distributions of $X$ and $Y$ are given by

$$
f\left(x \mid \sigma_{1}\right)=\frac{2}{\sigma_{1}} \frac{\exp \left\{-x / \sigma_{1}\right\}}{\left[1+\exp \left\{-x / \sigma_{1}\right\}\right]^{2}}, x \geq 0, \sigma_{1}>0,
$$

and

$$
f\left(y \mid \sigma_{2}\right)=\frac{2}{\sigma_{2}} \frac{\exp \left\{-y / \sigma_{2}\right\}}{\left[1+\exp \left\{-y / \sigma_{2}\right\}\right]^{2}}, y \geq 0, \sigma_{2}>0
$$

respectively.

Let $X_{1}, X_{2}, \cdots, X_{n_{1}}$ be a random sample of size $n_{1}$ from $\mathcal{H} \mathcal{L}\left(\sigma_{1}\right)$ and $Y_{1}, Y_{2}, \cdots, Y_{n_{2}}$ be a random sample of size $n_{2}$ from $\mathcal{H} \mathcal{L}\left(\sigma_{2}\right)$, respectively. Let $\mathbf{x}=\left(x_{1}, x_{2}, \cdots, x_{n_{1}}\right)$ be observations of $X_{1}, X_{2}, \cdots, X_{n_{1}}$ and $\mathbf{y}=\left(y_{1}, y_{2}, \cdots, y_{n_{2}}\right)$ be observations of $Y_{1}, Y_{2}, \cdots, Y_{n_{2}}$, respectively. We want to make a Bayesian inference about the ratio of scale parameters based on objective priors. 


\subsection{The probability matching priors}

For a prior $\pi$, let $\theta_{1}^{1-\alpha}(\pi ; \mathbf{X})$ denote the $(1-\alpha)$ th posterior quantile of $\theta_{1}$, that is,

$$
P^{\pi}\left[\theta_{1} \leq \theta_{1}^{1-\alpha}(\pi ; \mathbf{X}) \mid \mathbf{X}\right]=1-\alpha
$$

where $\boldsymbol{\theta}=\left(\theta_{1}, \cdots, \theta_{t}\right)^{T}$ and $\theta_{1}$ is the parameter of interest. We want to find priors $\pi$ for which

$$
P\left[\theta_{1} \leq \theta_{1}^{1-\alpha}(\pi ; \mathbf{X}) \mid \boldsymbol{\theta}\right]=1-\alpha+o\left(n^{-r}\right)
$$

for some $r>0$, as $n$ goes to infinity. Priors $\pi$ satisfying (2.4) are called matching priors. If $r=1 / 2$, then $\pi$ is referred to as a first order matching prior, while if $r=1, \pi$ is referred to as a second order matching prior.

In order to find such matching priors $\pi$, let

$$
\theta_{1}=\frac{\sigma_{2}}{\sigma_{1}} \text { and } \theta_{2}=\sigma_{1}^{n_{1}} \sigma_{2}^{n_{2}}
$$

With this parametrization, the likelihood function of parameters $\left(\theta_{1}, \theta_{2}\right)$ for the models $(2.1)$ and (2.2) is given by

$$
\begin{aligned}
& L\left(\theta_{1}, \theta_{2}\right) \\
& \propto \theta_{2}^{-1} \prod_{i=1}^{n_{1}}\left[1+\exp \left(-\theta_{1}^{\frac{n_{2}}{n_{1}+n_{2}}} \theta_{2}^{\frac{-1}{n_{1}+n_{2}}} x_{i}\right)\right]^{-2} \prod_{i=1}^{n_{2}}\left[1+\exp \left(-\theta_{1}^{\frac{-n_{1}}{n_{1}+n_{2}}} \theta_{2}^{\frac{-1}{n_{1}+n_{2}}} y_{i}\right)\right]^{-2} \\
& \times \exp \left\{-\theta_{1}^{\frac{n_{2}}{n_{1}+n_{2}}} \theta_{2}^{\frac{-1}{n_{1}+n_{2}}} \sum_{i=1}^{n_{1}} x_{i}-\theta_{1}^{\frac{-n_{1}}{n_{1}+n_{2}}} \theta_{2}^{\frac{-1}{n_{1}+n_{2}}} \sum_{i=1}^{n_{2}} y_{i}\right\}
\end{aligned}
$$

Based on (2.5), the Fisher information matrix is given by

$$
\mathbf{I}\left(\theta_{1}, \theta_{2}\right)=\left(\begin{array}{cc}
\frac{n_{1} n_{2}\left(3+\pi^{2}\right)}{9\left(n_{1}+n_{2}\right)} \theta_{1}^{-2} & 0 \\
0 & \frac{3+\pi^{2}}{9\left(n_{1}+n_{2}\right)^{2}} \theta_{2}^{-2}
\end{array}\right) .
$$

From the above Fisher information matrix $\mathbf{I}, \theta_{1}$ is orthogonal to $\theta_{2}$ in the sense of Cox and Reid (1987). Following Tibshirani (1989), the class of first order probability matching prior is characterized by

$$
\pi_{m}^{(1)}\left(\theta_{1}, \theta_{2}\right) \propto \theta_{1}^{-1} d\left(\theta_{2}\right)
$$

where $d\left(\theta_{2}\right)>0$ is an arbitrary function differentiable in its argument.

The class of prior given in (2.7) can be narrowed down to the second order probability matching priors as given in Mukerjee and Ghosh (1997). A second order probability matching prior is of the form (2.7), and $d$ must satisfy an additional differential equation (2.10) of Mukerjee and Ghosh (1997), namely

$$
\frac{1}{6} d\left(\theta_{2}\right) \frac{\partial}{\partial \theta_{1}}\left\{I_{11}^{-\frac{3}{2}} L_{1,1,1}\right\}+\frac{\partial}{\partial \theta_{2}}\left\{I_{11}^{-\frac{1}{2}} L_{112} I^{22} d\left(\theta_{2}\right)\right\}=0
$$


where

$$
\begin{aligned}
L_{1,1,1} & =E\left[\left(\frac{\partial \log L}{\partial \theta_{1}}\right)^{3}\right]=\frac{n_{1} n_{2}\left(n_{1}-n_{2}\right) \pi^{2}}{3\left(n_{1}+n_{2}\right)^{2}} \theta_{1}^{-3}, \\
L_{112} & =E\left[\frac{\partial^{3} \log L}{\partial \theta_{1}^{2} \partial \theta_{2}}\right]=\frac{n_{1} n_{2} \pi^{2}}{6\left(n_{1}+n_{2}\right)^{2}} \theta_{1}^{-2} \theta_{2}^{-1}, \\
I_{11} & =\frac{n_{1} n_{2}\left(3+\pi^{2}\right)}{9\left(n_{1}+n_{2}\right)} \theta_{1}^{-2}, I^{22}=\frac{9\left(n_{1}+n_{2}\right)^{2}}{3+\pi^{2}} \theta_{2}^{2} .
\end{aligned}
$$

Then (2.8) simplifies to

$$
\frac{\partial}{\partial \theta_{2}}\left\{\frac{\pi^{2}\left(n_{1} n_{2}\right)^{\frac{1}{2}}}{\left(3+\pi^{2}\right)^{\frac{3}{2}}\left(n_{1}+n_{2}\right)^{\frac{1}{2}}} \theta_{1}^{-1} \theta_{2} d\left(\theta_{2}\right)\right\}=0 .
$$

Hence the set of solution of (2.9) is of the form $d\left(\theta_{2}\right)=\theta_{2}^{-1}$. Thus the resulting second order probability matching prior is

$$
\pi_{m}^{(2)}\left(\theta_{1}, \theta_{2}\right) \propto \theta_{1}^{-1} \theta_{2}^{-1}
$$

Remark 2.1 There are alternative ways through which matching can be accomplished. Datta, Ghosh and Mukerjee (2000) provided a theorem which establishes the equivalence of second order matching priors and HPD matching priors (DiCiccio and Stern, 1994; Ghosh and Mukerjee, 1995) within the class of first order matching priors. The equivalence condition is that $I_{11}^{-3 / 2} L_{111}$ dose not depend on $\theta_{1}$. Since

$$
L_{111}=E\left[\frac{\partial^{3} \log L}{\partial \theta_{1}^{3}}\right]=\frac{n_{1} n_{2}\left[6\left(n_{1}+n_{2}\right)+\left(3 n_{1}+n_{2}\right) \pi^{2}\right]}{6\left(n_{1}+n_{2}\right)^{2}} \theta_{1}^{-3},
$$

$I_{11}^{-3 / 2} L_{111}$ does not depend on $\theta_{1}$. Therefore the second order probability matching prior (2.10) is a HPD matching prior. Also

$$
\begin{aligned}
& L_{11,1}=E\left[\frac{\partial^{2} \log L}{\partial \theta_{1}^{2}} \frac{\partial \log L}{\partial \theta_{1}}\right]=c_{1} \theta_{1}^{-3} \\
& L_{11,2}=E\left[\frac{\partial^{2} \log L}{\partial \theta_{1}^{2}} \frac{\partial \log L}{\partial \theta_{2}}\right]=c_{2} \theta_{1}^{-2} \theta_{2}^{-1}
\end{aligned}
$$

and $d\left(\theta_{2}\right)=\theta_{2}^{-1}$, where $c_{1}$ and $c_{2}$ are constants. Then

$$
\begin{aligned}
& \frac{\partial}{\partial \theta_{2}}\left\{L_{112} I^{22} I_{11}^{-1 / 2} d\left(\theta_{2}\right)\right\}=0, \frac{\partial}{\partial \theta_{2}}\left\{L_{11,2} I^{22} I_{11}^{-1 / 2} d\left(\theta_{2}\right)\right\}=0, \\
& \frac{\partial}{\partial \theta_{1}}\left\{I_{11}^{-3 / 2} L_{111}\right\}=0, \frac{\partial}{\partial \theta_{1}}\left\{I_{11}^{-3 / 2} L_{11,1}\right\}=0 .
\end{aligned}
$$

Therefore the second order matching prior (2.10) matches the alternative coverage probabilities (Mukerjee and Reid, 1999). 


\subsection{The reference priors}

Reference priors introduced by Bernardo (1979), and extended further by Berger and Bernardo (1992) have become very popular over the years for the development of noninformative priors. In this section, we derive the reference priors for different groups of ordering of $\left(\theta_{1}, \theta_{2}\right)$. Then due to the orthogonality of the parameters, following Datta and Ghosh (1995b), choosing rectangular compacts for each $\theta_{1}$ and $\theta_{2}$ when $\theta_{1}$ is the parameter of interest, the reference priors are given by as follows.

For the likelihood (2.5), if $\theta_{1}$ is the parameter of interest, then the reference prior distributions for group of ordering of $\left\{\left(\theta_{1}, \theta_{2}\right)\right\}$ is

$$
\pi_{1}\left(\theta_{1}, \theta_{2}\right) \propto \theta_{1}^{-1} \theta_{2}^{-1} .
$$

For group of ordering of $\left\{\theta_{1}, \theta_{2}\right\}$, the reference prior is

$$
\pi_{2}\left(\theta_{1}, \theta_{2}\right) \propto \theta_{1}^{-1} \theta_{2}^{-1} .
$$

Remark 2.2 From the above reference priors, we know that the one-at-a-time reference prior $\pi_{2}$ and Jeffreys' prior $\pi_{1}$ are the second order matching prior, and all priors are the same.

\section{Implementation of the Bayesian procedure}

We investigate the propriety of posteriors for a general class of priors which includes the reference prior and the matching prior. We consider the class of priors

$$
\pi\left(\theta_{1}, \theta_{2}\right) \propto \theta_{1}^{-a} \theta_{2}^{-b}
$$

where $a>0$ and $b>0$. The following general theorem can be proved.

Theorem 3.1 The posterior distribution of $\left(\theta_{1}, \theta_{2}\right)$ under the prior $\pi,(3.1)$, is proper if $b n_{1}-a+1>0$ and $b n_{2}+a-1>0$.

Proof: Note that the joint posterior for $\theta_{1}$ and $\theta_{2}$ given $\mathbf{x}$ and $\mathbf{y}$ is

$$
\begin{aligned}
& \pi\left(\theta_{1}, \theta_{2} \mid \mathbf{x}, \mathbf{y}\right) \\
& \propto \theta_{1}^{-a} \theta_{2}^{-b-1} \exp \left\{-\theta_{1}^{\frac{n_{2}}{n_{1}+n_{2}}} \theta_{2}^{\frac{-1}{n_{1}+n_{2}}} \sum_{i=1}^{n_{1}} x_{i}-\theta_{1}^{\frac{-n_{1}}{n_{1}+n_{2}}} \theta_{2}^{\frac{-1}{n_{1}+n_{2}}} \sum_{i=1}^{n_{2}} y_{i}\right\} \\
& \times \prod_{i=1}^{n_{1}}\left[1+\exp \left(-\theta_{1}^{\frac{n_{2}}{n_{1}+n_{2}}} \theta_{2}^{\frac{-1}{n_{1}+n_{2}}} x_{i}\right)\right]^{-2} \prod_{i=1}^{n_{2}}\left[1+\exp \left(-\theta_{1}^{\frac{-n_{1}}{n_{1}+n_{2}}} \theta_{2}^{\frac{-1}{n_{1}+n_{2}}} y_{i}\right)\right]^{-2}
\end{aligned}
$$

Then we get

$$
\begin{aligned}
\pi\left(\theta_{1}, \theta_{2} \mid \mathbf{x}, \mathbf{y}\right) & \leq \theta_{1}^{-a} \theta_{2}^{-b-1} \exp \left\{-\theta_{1}^{\frac{n_{2}}{n_{1}+n_{2}}} \theta_{2}^{\frac{-1}{n_{1}+n_{2}}} \sum_{i=1}^{n_{1}} x_{i}-\theta_{1}^{\frac{-n_{1}}{n_{1}+n_{2}}} \theta_{2}^{\frac{-1}{n_{1}+n_{2}}} \sum_{i=1}^{n_{2}} y_{i}\right\} \\
& \equiv \pi^{\prime}\left(\theta_{1}, \theta_{2} \mid \mathbf{x}, \mathbf{y}\right) .
\end{aligned}
$$


Thus integrating with respect to $\theta_{2}$ in $(3.3)$, we can get

$$
\begin{aligned}
\pi^{\prime}\left(\theta_{1} \mid \mathbf{x}, \mathbf{y}\right) & \propto \theta_{1}^{-b n_{2}-a}\left[\sum_{i=1}^{n_{1}} x_{i}+\theta_{1}^{-1} \sum_{i=1}^{n_{2}} y_{i}\right]^{-b\left(n_{1}+n_{2}\right)} \\
& =\left(\sum_{i=1}^{n_{1}} x_{i}\right)^{-b\left(n_{1}+n_{2}\right)} \int_{0}^{\infty} \theta_{1}^{-a+b n_{1}}\left(\theta_{1}+\frac{\sum_{j=1}^{n_{2}} y_{j}}{\sum_{i=1}^{n_{1}} x_{i}}\right)^{-b\left(n_{1}+n_{2}\right)} d \theta_{1}
\end{aligned}
$$

Letting $z=\theta_{1} /\left(\theta_{1}+k\right)$, where $k=\sum_{j=1}^{n_{2}} y_{j} / \sum_{i=1}^{n_{1}} x_{i}$, the above integration results in beta function. Thus the integration in (3.4) is finite if $b n_{1}-a+1>0$ and $b n_{2}+a-1>0$. This completes the proof.

Theorem 3.2 Under the prior (3.1), the marginal posterior density of $\theta_{1}$ is given by

$$
\begin{aligned}
& \pi\left(\theta_{1} \mid \mathbf{x}, \mathbf{y}\right) \\
& \propto \int_{0}^{\infty} \theta_{1}^{-a} \theta_{2}^{-b-1} \exp \left\{-\theta_{1}^{\frac{n_{2}}{n_{1}+n_{2}}} \theta_{2}^{\frac{-1}{n_{1}+n_{2}}} \sum_{i=1}^{n_{1}} x_{i}-\theta_{1}^{\frac{-n_{1}}{n_{1}+n_{2}}} \theta_{2}^{\frac{-1}{n_{1}+n_{2}}} \sum_{i=1}^{n_{2}} y_{i}\right\} \\
& \times \prod_{i=1}^{n_{1}}\left[1+\exp \left(-\theta_{1}^{\frac{n_{2}}{n_{1}+n_{2}}} \theta_{2}^{\frac{-1}{n_{1}+n_{2}}} x_{i}\right)\right]^{-2} \prod_{i=1}^{n_{2}}\left[1+\exp \left(-\theta_{1}^{\frac{-n_{1}}{n_{1}+n_{2}}} \theta_{2}^{\frac{-1}{n_{1}+n_{2}}} y_{i}\right)\right]^{-2} d \theta_{2} .
\end{aligned}
$$

Therefore we have the marginal posterior density of $\theta_{1}$, and so it is easy to compute the marginal moment of $\theta_{1}$. In Section 4 , we investigate the frequentist coverage probabilities for the reference prior.

\section{Numerical studies}

We evaluate the frequentist coverage probability by investigating the credible interval of the marginal posterior density of $\theta_{1}$ under the reference prior given in Section 3 for several configurations $\left(\sigma_{1}, \sigma_{2}\right)$ and $\left(n_{1}, n_{2}\right)$. That is to say, the frequentist coverage of a $(1-\alpha)$ th posterior quantile should be close to $1-\alpha$. This is done numerically. Table 4.1 gives numerical values of the frequentist coverage probabilities of 0.05 (0.95) posterior quantiles for the our prior. The computation of these numerical values is based on the following algorithm for any fixed true $\left(\lambda_{1}, \lambda_{2}\right)$ and any prespecified probability value $\alpha$. Here $\alpha$ is $0.05(0.95)$. Let $\theta_{1}^{\pi}(\alpha \mid \mathbf{X}, \mathbf{Y})$ be the posterior $\alpha$-quantile of $\theta_{1}$ given $\mathbf{X}$ and $\mathbf{Y}$. That is, $F\left(\theta_{1}^{\pi}(\alpha \mid \mathbf{X}, \mathbf{Y}) \mid \mathbf{X}, \mathbf{Y}\right)=\alpha$, where $F(\cdot \mid \mathbf{X}, \mathbf{Y})$ is the marginal posterior distribution of $\theta_{1}$. Then the frequentist coverage probability of this one sided credible interval of $\theta_{1}$ is

$$
P_{\left(\theta_{1}, \theta_{2}\right)}\left(\alpha ; \theta_{1}\right)=P_{\left(\theta_{1}, \theta_{2}\right)}\left(0<\theta_{1} \leq \theta_{1}^{\pi}(\alpha \mid \mathbf{X}, \mathbf{Y})\right) .
$$

The computed $P_{\left(\theta_{1}, \theta_{2}\right)}\left(\alpha ; \theta_{1}\right)$ when $\alpha=0.05(0.95)$ is shown in Table 4.1. In particular, for fixed $n$ and $\left(\sigma_{1}, \sigma_{2}\right)$, we take 10,000 independent random samples of $\mathbf{X}=\left(X_{1}, \cdots, X_{n_{1}}\right)$ and $\mathbf{Y}=\left(Y_{1}, \cdots, Y_{n_{2}}\right)$ from the half logistic distributions, respectively.

In Table 4.1, we can observe that the reference prior meets very well the target coverage probabilities even for the small sample sizes. Also note that the results of table are not much sensitive to the change of the values of $\left(\theta_{1}, \sigma_{1}\right)$. Thus we recommend to use the reference prior. 
Table 4.1 Frequentist coverage probability of $0.05(0.95)$ posterior quantiles of $\theta_{1}$

\begin{tabular}{|c|c|c|c|c|}
\hline$\theta_{1}$ & $\sigma_{1}$ & $n_{1}$ & $n_{2}$ & $\pi_{r}$ \\
\hline \multirow{12}{*}{0.1} & \multirow{4}{*}{0.1} & 5 & 5 & $0.048(0.951)$ \\
\hline & & 5 & 10 & $0.053(0.949)$ \\
\hline & & 10 & 10 & $0.053(0.949)$ \\
\hline & & 10 & 15 & $0.054(0.950)$ \\
\hline & \multirow{4}{*}{1.0} & 5 & 5 & $0.044(0.945)$ \\
\hline & & 5 & 10 & $0.050(0.948)$ \\
\hline & & 10 & 10 & $0.052(0.952)$ \\
\hline & & 10 & 15 & $0.046(0.949)$ \\
\hline & \multirow{4}{*}{10.0} & 5 & 5 & $0.049(0.947)$ \\
\hline & & 5 & 10 & $0.051(0.953)$ \\
\hline & & 10 & 10 & $0.051(0.949)$ \\
\hline & & 10 & 15 & $0.049(0.951)$ \\
\hline \multirow{12}{*}{1.0} & \multirow{4}{*}{0.1} & 5 & 5 & $0.051(0.949)$ \\
\hline & & 5 & 10 & $0.048(0.953)$ \\
\hline & & 10 & 10 & $0.048(0.948)$ \\
\hline & & 10 & 15 & $0.051(0.948)$ \\
\hline & \multirow{4}{*}{1.0} & 5 & 5 & $0.050(0.948)$ \\
\hline & & 5 & 10 & $0.052(0.951)$ \\
\hline & & 10 & 10 & $0.048(0.952)$ \\
\hline & & 10 & 15 & $0.049(0.950)$ \\
\hline & \multirow{4}{*}{10.0} & 5 & 5 & $0.049(0.949)$ \\
\hline & & 5 & 10 & $0.046(0.950)$ \\
\hline & & 10 & 10 & $0.046(0.950)$ \\
\hline & & 10 & 15 & $0.047(0.949)$ \\
\hline \multirow{12}{*}{10.0} & \multirow{4}{*}{0.1} & 5 & 5 & $0.047(0.951)$ \\
\hline & & 5 & 10 & $0.052(0.949)$ \\
\hline & & 10 & 10 & $0.046(0.951)$ \\
\hline & & 10 & 15 & $0.051(0.949)$ \\
\hline & \multirow{4}{*}{1.0} & 5 & 5 & $0.050(0.951)$ \\
\hline & & 5 & 10 & $0.052(0.947)$ \\
\hline & & 10 & 10 & $0.046(0.947)$ \\
\hline & & 10 & 15 & $0.049(0.947)$ \\
\hline & \multirow{4}{*}{10.0} & 5 & 5 & $0.043(0.950)$ \\
\hline & & 5 & 10 & $0.049(0.948)$ \\
\hline & & 10 & 10 & $0.052(0.950)$ \\
\hline & & 10 & 15 & $0.051(0.951)$ \\
\hline
\end{tabular}

Example 4.1 This example is taken from Balakrishnan and Puthenpura (1986). The data is failure times, in minutes, for a specific type of electrical insulation in an experiment in which the insulation was subjected to a continuously increasing voltage stress. For this data, Balakrishnan and Puthenpura (1986) concluded that the half logistic distribution fits the data better than an exponential distribution. For estimation of ratio of scale parameters, we randomly divided this data into two groups. The data sets are given by

Group 1: 21.8, 70.7, 151.9, 75.3, 12.3, 28.6.

Group 2: 24.4, 138.6, 95.5, 98.1, 43.2, 46.9 .

For this data, the maximum likelihood estimate (MLE) of $\theta_{1}$ is 1.1826 and the corresponding $95 \%$ asymptotic confidence interval of $\theta_{1}$ is $(0.0635,2.3016)$. Bayes estimate and the $95 \%$ credible interval based on the reference prior are 1.3431 and $(0.4384,3.1832)$, respectively. The estimate based on the reference prior and the MLE give almost same results, but the confidence intervals based on the MLE and the reference prior give some different results. 


\section{Concluding remarks}

In the half logistic models, we have found the second order matching prior and the reference prior for the ratio of the scale parameters. We revealed that the second order matching prior is a HPD matching prior and matches the alternative coverage probabilities up to the second order. It turns out that the reference prior and Jeffreys' prior are the second order matching prior. As illustrated in our numerical study, the reference prior meets very well the target coverage probabilities. Thus we recommend the use of the reference prior for Bayesian inference of the ratio of the scale parameters in two independent half logistic distributions.

\section{References}

Adatia, A. (1997). Approximate BLUEs of the parameters of the half logistic distribution based on fairly large doubly censored samples. Computational Statistics and Data Analysis, 24, 179-191.

Balakrishnan, N. (1985). Order statistics from the half logistic distribution. Journal of Statistical Computation and Simulation, 20, 287-309.

Balakrishnan, N. and Puthenpura, S. (1986). Best linear unbiased estimators of location and scale parameters of the half logistic distribution. Journal of Statistical Computation and Simulation, 25, $193-204$.

Balakrishnan, N. and Wong, K. H. T. (1991). Approximate MLEs for the location and scale parameters of the half logistic distribution with type-II right censoring. IEEE Transactions on Reliability, 40, $140-145$.

Berger, J. O. and Bernardo, J. M. (1989). Estimating a product of means : Bayesian analysis with reference priors. Journal of the American Statistical Association, 84, 200-207.

Berger, J. O. and Bernardo, J. M. (1992). On the development of reference priors (with discussion). In Bayesian Statistics IV, edited by J. M. Bernardo, et al., Oxford University Press, Oxford, 35-60.

Bernardo, J. M. (1979). Reference posterior distributions for Bayesian inference (with discussion). Journal of Royal Statistical Society B, 41, 113-147.

Cox, D. R. and Reid, N. (1987). Orthogonal parameters and approximate conditional inference (with discussion). Journal of Royal Statistical Society B, 49, 1-39.

Datta, G. S. and Ghosh, J. K. (1995a). On priors providing frequentist validity for Bayesian inference. Biometrika, 82, 37-45.

Datta, G. S. and Ghosh, M. (1995b). Some remarks on noninformative priors. Journal of the American Statistical Association, 90, 1357-1363.

Datta, G. S. and Ghosh, M. (1996). On the invariance of noninformative priors. The Annal of Statistics, 24, 141-159.

Datta, G. S., Ghosh, M. and Mukerjee, R. (2000). Some new results on probability matching priors. Calcutta Statistical Association Bulletin, 50, 179-192.

DiCiccio, T. J. and Stern, S. E. (1994). Frequentist and Bayesian Bartlett correction of test statistics based on adjusted profile likelihood. Journal of Royal Statistical Society B, 56, 397-408.

Ghosh, J. K. and Mukerjee, R. (1992). Noninformative priors (with discussion). In Bayesian Statistics IV, edited by J. M. Bernardo, et al., Oxford University Press, Oxford, 195-210.

Ghosh, J. K. and Mukerjee, R. (1995). Frequentist validity of highest posterior density regions in the presence of nuisance parameters. Statistics \& Decisions, 13, 131-139.

Kang, S. B. and Park, Y. K. (2005). Estimation for the half-logistic distribution based on multiply type-II censored samples. Journal of the Korean Data \& Information Science Society, 16, 145-156.

Kang, S. G. (2011). Noninformative priors for the common mean in log-normal distributions. Journal of the Korean Data \& Information Science Society, 22, 1241-1250.

Kim, C. and Han, K. (2010). Estimation of the scale parameter of the half-logistic distribution under progressively type-II censored sample. Statistical Papers, 51, 375-387.

Kim, D. H., Kang, S. G. and Lee, W. D. (2009). Noninformative priors for Pareto distribution. Journal of the Korean Data \& Information Science Society, 20, 1213-1223.

Mukerjee, R. and Dey, D.K. (1993). Frequentist validity of posterior quantiles in the presence of a nuisance parameter : Higher order asymptotics. Biometrika, 80, 499-505.

Mukerjee, R. and Ghosh, M. (1997). Second order probability matching priors. Biometrika, 84, 970-975. 
Mukerjee, R. and Reid, N. (1999). On a property of probability matching priors: matching the alternative coverage probabilities. Biometrika, 86, 333-340.

Stein, C. (1985). On the coverage probability of confidence sets based on a prior distribution. Sequential Methods in Statistics, Banach Center Publications, 16, 485-514.

Tibshirani, R. (1989). Noninformative priors for one parameter of many. Biometrika, 76, 604-608.

Welch, B. L. and Peers, H. W. (1963). On formulae for confidence points based on integrals of weighted likelihood. Journal of Royal Statistical Society B, 25, 318-329. 